\title{
Applications of X-ray Powder Diffraction in Pharmaceutical Industry
}

Rajni M. Bhardwaj,

Small Molecule Design and Development, Eli Lilly \& Company, Indianapolis, IN 46285, USA

Experimental screening is often used to explore the range of possible solid forms (polymorphs, hydrate/ solvates, salts, co-crystals, amorphous) of pharmaceuticals. The properties of two solid forms of a same drug can vary substantially with potentially significant impact on solubility, dissolution, bioavailability, stability, processability, and shelf life. Hence, for any candidate molecule, it is critical to have an understanding of the relevant solid forms and their relationships so that appropriate form can be taken forward for commercialization. X-ray powder diffraction (XRPD) is utilized as a primary characterization tool during solid form discovery and drug development cycle to ensure reproducibility of the selected form. Various case studies demonstrating utilization of XRPD as a primary tool in solid form discovery and crystal structure determination (Structure Determination from Powder Data, SDPD) of samples where suitable single crystals could not be obtained, using global optimization approaches such as simulated annealing will be discussed. In case of salt-co-crystals, it is regulatory requirement to provide evidence to confirm the proton position. A complementary approach utilizing SDPD and DFT calculations for confirmation of salt vs. co-crystal will also be demonstrated. In addition, case studies where XRPD has been utilized to have better understanding of structure property relationships, identifying structural model from crystal structure prediction for crystal structure determination/refinement and phase transformation during processing and storage will be presented. In another unique application of XRPD to large molecules, solid state form change was confirmed using XRPD following a manufacturing process change. These results helped in altering the process change to produce the desired form. 